\title{
A meta-heuristic cuckoo search and eigen permutation approach for model order reduction
}

\author{
AKHILESH KUMAR GUPTA*, DEEPAK KUMAR and PAULSON SAMUEL \\ Motilal Nehru National Institute of Technology, Allahabad 211004, India \\ e-mail: akhileshgupta08@gmail.com; deepak_kumar@mnnit.ac.in; paul@mnnit.ac.in
}

MS received 25 August 2017; revised 29 November 2017; accepted 2 December 2017; published online 17 April 2018

\begin{abstract}
In this paper, a new model order reduction technique is presented by combining the benefits of the meta-heuristic cuckoo search optimization and Eigen permutation methods for order reduction of higher order continuous-time systems. In the proposed approach, the numerator and the denominator polynomials of reduced order model are determined by Cuckoo search and Eigen permutation approaches, respectively. The proposed approach preserves the stability of the original system into the lower order model as the Eigen permutation retains the dominant pole with simultaneous cluster formation of the remaining real and complex poles. The effectiveness of the proposed method is validated by single-input single-output and multiple-inputs multipleoutputs numerical examples.
\end{abstract}

Keywords. Cuckoo search; Eigen permutation; Lévy flight; model order reduction; MOR.

\section{Introduction}

The real-life problems are quite difficult for the analysis, design and numerical simulation as it results in a complex higher order system. Model order reduction (MOR) is the problem of reducing the order of a complex higher order system (HOS) into a simplified lower order model of reasonable accuracy by retaining the fundamental properties such as stability, steady-state error, transient response and so on. Some of the classical MOR techniques are continued fraction expansion (CFE) methods [1-3], Padè approximation [4] and moment matching technique [5]. However, instability problem arises with these methods in some cases [6]. To overcome this severe drawback, several stability preservation methods such as dominant modes retention [7], factor division [8], Mihailov criterion [9], Routh approximation [10], Hurwitz polynomial approximation [11, 12], stability equation method [13] and differentiation method [14] are presented. Later on, some mixed techniques [15-23] are developed in which numerator coefficients are derived by a classical MOR/optimization method and the denominator polynomials of reduced order model (ROM) are determined by a stability preservation technique [8-13] to ensure the stability of ROM.

Wilson [24, 25] discussed the issue of optimality in MOR and suggested an optimization technique based on error minimization. Subsequently, many researchers used natureinspired optimization techniques such as genetic algorithm [26, 27], harmony search [28], particle swarm optimization

*For correspondence
[16, 29-31], big bang-big crunch optimization [32-35], cuckoo search $[18,36]$ and so on for MOR of complex systems. Some improvements of the cuckoo search method are presented in [37, 38]. Long et al [37] proposed a hybrid CS algorithm based on the Solis and Wets local search technique for constrained global optimization. Further, the hybridization of genetic algorithm and CS approach is proposed to make a balance between exploration and exploitation ability [38]. Furthermore, modified cuckoo search (MCS) is also applied in MOR field [39-41]. The meta-heuristic approaches have strongly proved their capability and effectiveness in MOR field. Recently, various MOR techniques based on optimization approaches are proposed by several authors. These algorithms minimize the fitness functions which are usually integral absolute error (IAE) or integral square error (ISE) to determine the reduced order parameters.

The complexity and convergence speed of an optimization algorithm is dependent on the number of unknown variables to be optimized and the selection range of the respective variables. Therefore, it is a time-consuming process to reduce the higher order complex system into a lower order model with a significant number of unknown variables by using optimization method for both numerator and denominator polynomials. Further, an optimization technique does not ensure the stability of a ROM and might result in an unstable ROM if applied to the denominator of the HOS without proper selection of range.

Therefore, to deal with these issues, a new mixed method for order reduction is proposed by combining the Eigen permutation approach (EP) [42] and cuckoo search (CS) 
optimization technique $[43,44]$. The zeros and poles of the proposed ROM are determined by cuckoo search and Eigen permutation techniques, respectively. The proposed method requires a lower number of unknown variables to be optimized which reduces the effort of the cuckoo search optimization towards a solution and range selection for obtaining the ROM. Equality of stiffness for original and reduced system generates non-minimum phase response in some cases. The proposed approach has a unique feature to solve this issue. The proposed method results in stable reduced models for a stable higher order system.

The paper is organized as follows. The problem statement is narrated in section 2. The concepts of Eigen permutation and cuckoo search optimization are discussed in section 3 . The results obtained with the proposed method for numerical examples are given in section 4. Further, the comparison with the well-known methods through performance indices, step responses and modeling error curves are also given in section 5 . Finally, conclusive remarks are presented in section 6.

\section{Problem statement}

The objective of the paper is to obtain a ROM from a given continuous-time single-input single-output and (SISO) and multiple-inputs multiple-outputs (MIMO) systems to provide ease in numerical simulation. A SISO system is a simple system with single-input, single-output and the output are controlled by one control signal. On the other hand, MIMO is a comparatively complex system with the multiple inputs-multiple outputs.

Consider $n^{\text {th }}$ order transfer function of a SISO system as

$$
\begin{aligned}
T_{n}(s) & =\frac{N_{n}(s)}{D_{n}(s)}=\frac{c_{n-1} s^{n-1}+c_{n-2} s^{n-2}+\cdots+c_{1} s+c_{0}}{d_{n} s^{n}+d_{n-1} s^{n-1}+\cdots+d_{1} s+d_{0}} \\
& =\frac{\sum_{i=0}^{n-1} c_{i} s^{i}}{\sum_{i=0}^{n} d_{i} s^{i}}
\end{aligned}
$$

where $c_{i}$ 's and $d_{i}$ 's are the numerator and denominator polynomial coefficients of the higher order system, respectively.

Here, the objective is to find an $r^{\text {th }}(r<n)$ order ROM with the following transfer function

$$
\begin{aligned}
T_{r}(s)= & \frac{N_{r}(s)}{D_{r}(s)}=\frac{l_{r-1} s^{r-1}+l_{r-2} s^{r-2}+\cdots \cdots+l_{1} s+l_{0}}{k_{r} s^{r}+k_{r-1} s^{r-1}+\cdots \cdots+k_{1} s+k_{0}} \\
= & \frac{\sum_{i=0}^{r-1} l_{i} s^{i}}{\sum_{i=0}^{r} k_{i} s^{i}}
\end{aligned}
$$

where $k_{i}$ 's and $l_{i}$ 's are the denominator and numerator polynomial coefficients of the ROM, respectively.

The $n^{\text {th }}$ order multiple-input multiple-output (MIMO) system with $x$ inputs and $y$ outputs can be defined by the transfer function matrix (TFM) given below:

$$
\left[T_{n}(s)\right]=\frac{1}{D_{n}(s)}\left[\begin{array}{ccccc}
\alpha_{11}(s) & \alpha_{12}(s) & \alpha_{13}(s) & \ldots & \alpha_{1 x}(s) \\
\alpha_{21}(s) & \alpha_{22}(s) & \alpha_{23}(s) & \ldots & \alpha_{2 x}(s) \\
\vdots & \vdots & \vdots & \ldots & \vdots \\
\alpha_{y 1}(s) & \alpha_{y 2}(s) & \alpha_{y 3}(s) & \ldots & \alpha_{y x}(s)
\end{array}\right]
$$

The generalized transfer function of the above HOS is written as

$$
\begin{aligned}
& {\left[T_{n}(s)\right]=\left[t_{i j}(s)\right], \quad i=1,2, \ldots y \quad \& \quad j=1,2, \ldots x} \\
& =\frac{\alpha_{i j}(s)}{D_{n}(s)}=\frac{\alpha_{n-1} s^{n-1}+\alpha_{n-2} s^{n-2}+\cdots+\alpha_{1} s+\alpha_{0}}{\beta_{n} s^{n}+\beta_{n-1} s^{n-1}+\cdots+\beta_{1} s+\beta_{0}}
\end{aligned}
$$

Now, the TFM of the reduced model with $x$ inputs and $y$ outputs are given as

$$
\left[T_{r}(s)\right]=\left[\begin{array}{ccccc}
\gamma_{11}(s) & \gamma_{12}(s) & \gamma_{13}(s) & \ldots & \gamma_{1 x}(s) \\
\gamma_{21}(s) & \gamma_{22}(s) & \gamma_{23}(s) & \ldots & \gamma_{2 x}(s) \\
\vdots & \vdots & \vdots & \ldots & \vdots \\
\gamma_{y 1}(s) & \gamma_{y 2}(s) & \gamma_{y 3}(s) & \ldots & \gamma_{y x}(s)
\end{array}\right]
$$

The generalized transfer function of $r^{\text {th }}$ order reduced model can be represented as

$$
\begin{aligned}
{\left[T_{r}(s)\right] } & =\left[r_{i j}(s)\right], \quad i=1,2, \ldots y \quad \& \quad j=1,2, \ldots x \\
& =\frac{\gamma_{i j}(s)}{D_{r}(s)}=\frac{\gamma_{r-1} s^{r-1}+\gamma_{r-2} s^{r-2}+\cdots+\gamma_{1} s+\gamma_{0}}{\delta_{r} s^{r}+\delta_{r-1} s^{r-1}+\cdots+\delta_{1} s+\delta_{0}}
\end{aligned}
$$

\section{Proposed technique}

In this section, a novel mixed method is proposed by integrating the attributes of CS and EP techniques for the simplification of continuous-time systems. In this approach, the poles of ROM are determined by the Eigen permutation technique whereas the cuckoo search optimization method is adopted for the calculation of numerator polynomial.

\subsection{Eigen permutation approach}

Eigen permutation algorithm [42] is based on retaining the most dominant eigen value of the original system into the resulting ROM. It is a simple, efficient and computer- 
oriented algorithm and also deals with the imaginary roots of the HOS. The procedure of modified EP to determine the denominator polynomial is summarized in the following steps:

Step 1: [Clustering] Make the clusters of real and complex poles separately located in left s plane.

Step 2: [Sorting and retention] Sort the poles in ascending order with retention of the most dominant pole.

Step 3: [Repetition] Check whether the real parts of the poles are repeated or not:

Case 1) If a pole is repeated, then

$$
\begin{array}{r}
a_{e i}=\left|a_{1}\right|, \quad i=1,2, \ldots, r \text { for real roots, } \\
\operatorname{Re}\left(\sigma_{e 1}\right)=\operatorname{Re}\left(\sigma_{1}\right) \quad \text { for complex roots, }
\end{array}
$$

where $a_{1}, a_{2}, \ldots \ldots \ldots, a_{n}$ and $\left(\operatorname{Re}\left(\sigma_{1}\right) \pm j \operatorname{Im}\left(\sigma_{1}\right)\right), \ldots$, $\left(\operatorname{Re}\left(\sigma_{m}\right) \pm j \operatorname{Im}\left(\sigma_{m}\right)\right)$ are real and/or complex conjugate poles of the original model.

Case 2) If the real parts of the poles are not repeated, the pole next to a dominant pole is determined as below.

For real poles:

$$
\begin{aligned}
a_{e z} & =\frac{1}{\operatorname{Re}\left(a_{n}\right)}\left[\frac{\sum_{k=1}^{n} \operatorname{Re}\left(\mathrm{a}_{\mathrm{k}}\right)}{z-1}\right], \quad k=1,2, \ldots, n ; \text { and } \\
z & =2,3, \ldots r
\end{aligned}
$$

For complex poles:

Further, the real and imaginary part of the complex roots are obtained as

$$
\begin{aligned}
& \operatorname{Re}\left(\sigma_{e z}\right)=\frac{1}{\operatorname{Re}\left(\sigma_{m}\right)}\left[\frac{\sum_{k=1}^{m} \operatorname{Re}\left(\sigma_{\mathrm{k}}\right)}{z-1}\right], \quad k=1,2, \ldots, m ; \\
& \text { and } z=2,3, \ldots d
\end{aligned}
$$$$
\begin{gathered}
\operatorname{Im} \sigma_{e z}=\frac{1}{2 \operatorname{Im}\left(\sigma_{m}\right)}\left[\frac{\sum_{k=1}^{m} \operatorname{Im}\left(\sigma_{\mathrm{k}}\right)}{z-1}\right], \quad k=1,2, \ldots, m \quad \text { and } \\
z=2,3, \ldots d
\end{gathered}
$$

where $a_{e 1}, a_{e 2}, \ldots, a_{e r}$ and $\left(\operatorname{Re}\left(\sigma_{e 1}\right) \pm j \operatorname{Im}\left(\sigma_{e 1}\right)\right), \ldots$, $\left(\operatorname{Re}\left(\sigma_{e d}\right) \pm j \operatorname{Im}\left(\sigma_{e d}\right)\right)$ are real and/or complex conjugate poles of the reduced model

Step 4: [Computation] Compute the polynomial corresponding to real and complex roots as

$$
D_{r}(s)=\left(s-a_{e 1}\right)\left(s-a_{e 2}\right) \ldots\left(s-a_{e r}\right)
$$

$$
\begin{aligned}
D_{r}(s)= & \left(s-\left(\operatorname{Re}\left(\sigma_{e 1}\right)+j \operatorname{Im}\left(\sigma_{e 1}\right)\right)\right) \\
& \left(s-\left(\operatorname{Re}\left(\sigma_{e 1}\right)-j \operatorname{Im}\left(\sigma_{e 1}\right)\right)\right) \ldots \\
& \ldots\left(s-\left(\operatorname{Re}\left(\sigma_{e d}\right)+j \operatorname{Im}\left(\sigma_{e d}\right)\right)\right) \\
& \left(s-\left(\operatorname{Re}\left(\sigma_{e d}\right)-j \operatorname{Im}\left(\sigma_{e d}\right)\right)\right)
\end{aligned}
$$

Finally, the desired reduced order denominator polynomial $D_{r}(s)$ is obtained from the Eq. (11) and/or (12).

\subsection{Cuckoo search algorithm}

In this section, CS optimization approach is discussed which is used to determine the numerator polynomial of ROM. Yang and Deb [36, 44] developed cuckoo search (CS) in which the invasive breeding strategy of fascinating bird cuckoos is utilized. Cuckoos lay their eggs in other host birds to fertilize their eggs. If the host bird identifies cuckoos' eggs, the host bird either throws it away or changes its nest for a new one. In the CS algorithm, each egg in the nest is an emblem of the solution, whereas a cuckoo egg depicts a unique solution. Whenever an improved solution is obtained, previous one is replaced with the new solution. Further, many animals and insects, even light also demonstrate the typical features of Lévy flight in their flight behaviour. Lévy flights are just a random walk whose flight step length is dependent on a power law formula often called Lévy distribution. Three rules which explain the natural behaviour of cuckoos for the CS optimization algorithm are as follows:

1. Every nest is selected randomly, and every cuckoo dumps own laid eggs in it at a time.

2. The best nest with a sublime quality of egg goes to the next generations.

3. If the host bird recognizes the alien egg from the fixed number of nests with probability $\mathrm{p}_{\mathrm{r}} \in(0,1)$, it either kicks off the foreigner egg or leave the own nest and builds a new nest at the new location.

Furthermore, the last assumption is merely a replacement of the $n$ host by new nest with a fraction of $p_{\mathrm{r}}$ (with new random solutions). For an optimization problem, the science of a solution is directly dependent on the value of its objective function or fitness function. In this paper, integral absolute error (IAE) is used as a fitness function for Examples 1 and 2 whereas Examples 3 and 4 are solved by minimization of integral square error (ISE) as the fitness function. The IAE and ISE are expressed by

$$
\begin{aligned}
& f i=\sum_{j=1}^{N}|y(j \Delta t)-y r(j \Delta t)| \\
& f i=\sum_{j=1}^{N}[y(j \Delta t)-y r(j \Delta t)]^{2}
\end{aligned}
$$




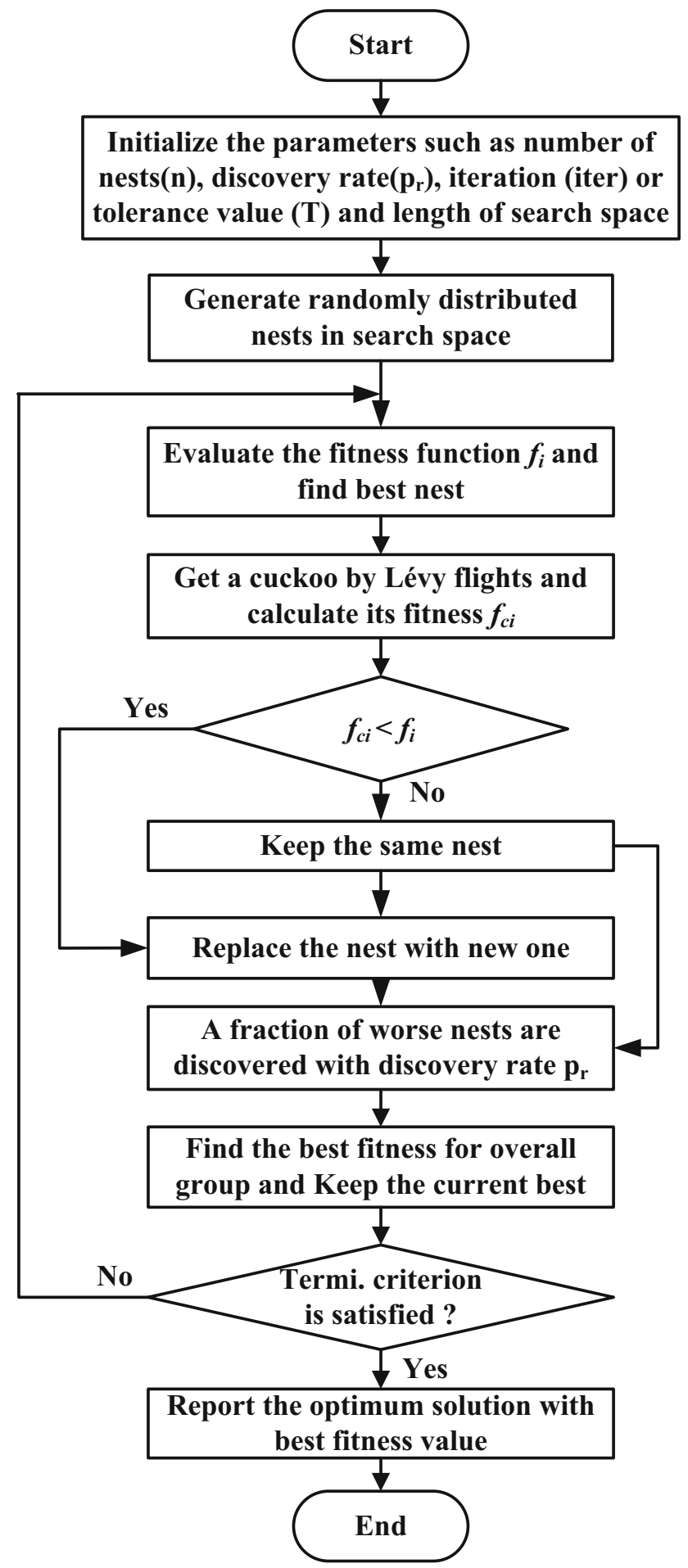

Figure 1. Flow chart of cuckoo search algorithm.

where $N=\frac{T_{s}}{\Delta t} ; y(j \Delta t)$ and $y_{r}(j \Delta t)$ are the unit step responses of the higher order and reduced order model at $t=\Delta t$. Here, the interval of the proposed approach is selected as $\left[0, T_{s}\right]$ for the minimization of fitness function where $T_{s}$ is the setting time. The unit step response is used for the minimization of the objective function as it gives necessary information of a system such as transient and steady-state behaviour.

Based on the above-described rules, the fundamental steps of the CS optimization algorithm are summarized as below:

Step 1: [Declaration] Define the CS parameters such as decision variables with the lower and upper boundaries, number of nests, discovery rates, number of iterations or termination criterion and objective function.

Step 2: [nitialization] Initialize the randomly generated nests.

Step 3: [Comparison and Selection] The nest or solution with lowest fitness value is chosen as a best-suited nest. Step 4: [Lévy flight] A new solution for a chosen cuckoo is performed by Lévy flight as given below

$$
K_{i}^{t+1}=K_{i}+\alpha \otimes \operatorname{Levy}(\lambda)
$$

where $\alpha$ is the step size $(\alpha>0)$ and the symbol $\otimes$ is an entry-wise multiplication. Lévy distribution function determines the random step size as

$$
\text { Lévy } \sim u=t^{-\lambda} ;(1<\lambda<3)
$$

which is the infinite variance and infinite mean. Lévy distribution is based on the law of "power law step length distribution". The random Lévy flight algorithm is a calculation of the fitness value of the cuckoos and replacement of chosen nest with a new one if the fitness value of chosen nest is worse than that of a cuckoo by Lévy flights. Step 5: [Best fitness] Find the lowest fitness value of the whole group

Step 6: [Iteration] Go to step 4 until the maximum number of iterations is reached, or stopping criterion is met. Display the lowest fitness value and corresponding optimum solution. The flow chart of cuckoo search is shown in figure 1.

\section{Numerical examples}

Example 1: Consider the fourth order transfer function [32-34, 43] given as

$$
\mathrm{T}_{4}(\mathrm{~s})=\frac{\mathrm{s}^{3}+7 \mathrm{~s}^{2}+24 \mathrm{~s}+24}{\mathrm{~s}^{4}+10 \mathrm{~s}^{3}+35 \mathrm{~s}^{2}+50 \mathrm{~s}+24}
$$

\subsection{Determination of denominator polynomial}

The poles of the above transfer function are $a_{1}=-1.00$, $a_{2}=-2.00, a_{3}=-3.00, a_{4}=-4.00$. Now the poles of the second order reduced model are obtained by the Eigen permutation which is $a_{e 1}=-1.00, a_{e 2}=-2.50$. Hence, denominator polynomial of reduced model is

$$
D_{2}(s)=s^{2}+3.5 s+2.5
$$




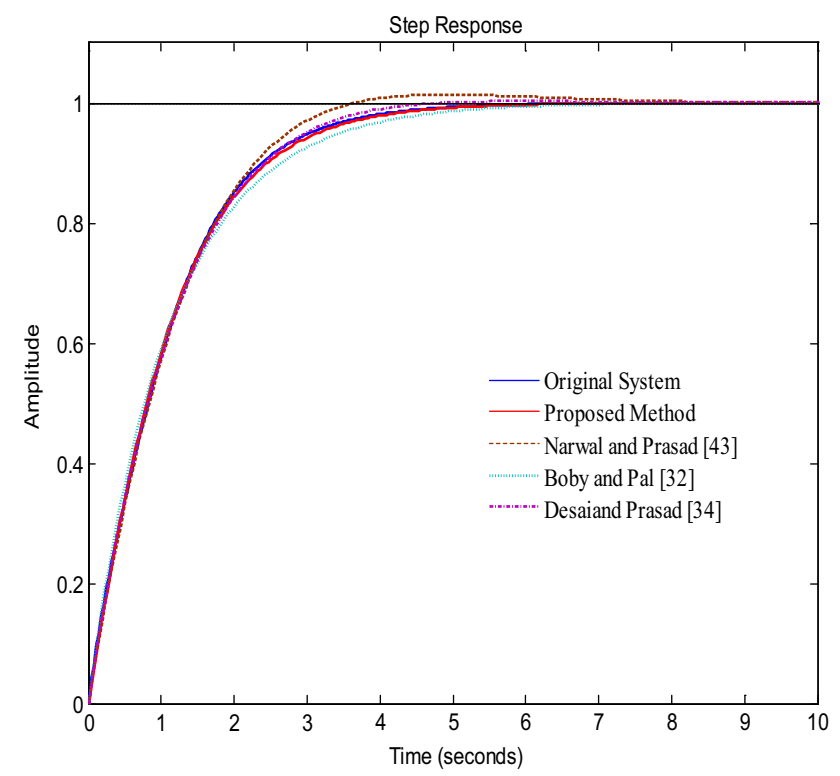

Figure 2. Step responses to different methods for Example 1.

\subsection{Determination of numerator polynomial}

The coefficients of the numerator polynomial are determined by CS optimization algorithm with population size $n=25$, discovery rate $p_{r}=0.25$ and iteration value iter $=100$. A suitable multiplying factor is multiplied by

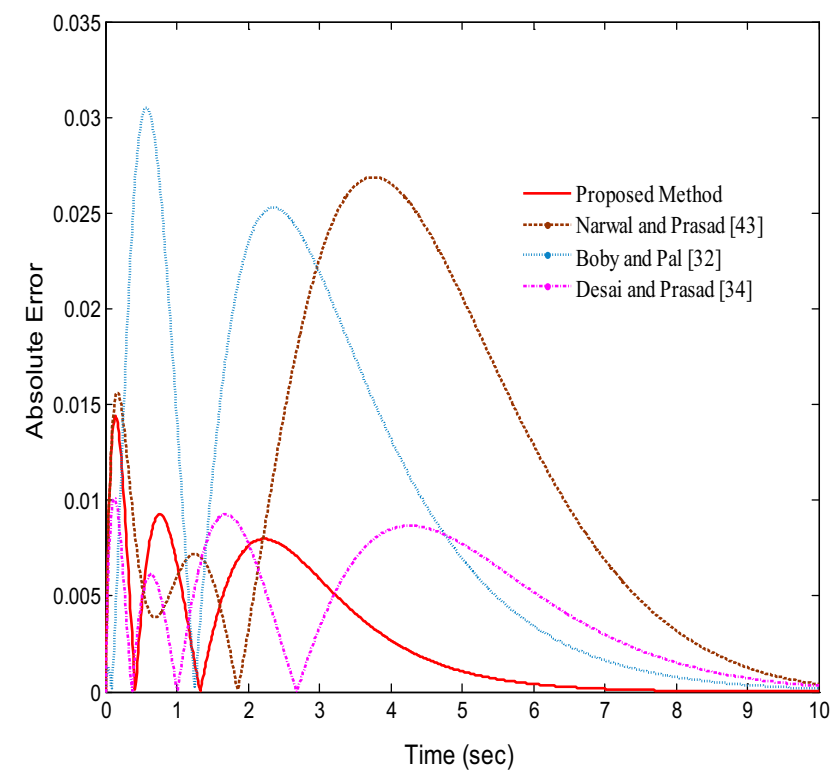

Figure 3. Plots of absolute error obtained by various methods for Example 1.

The superiority of the proposed method over other methods can be easily be observed from the table 2 .

Example 2: Consider the transfer function of eighth order system $[42,48]$ as

$$
T_{8}(s)=\frac{18 s^{7}+514 s^{6}+5982 s^{5}+36380 s^{4}+122664 s^{3}+222088 s^{2}+185760 s+40320}{s^{8}+36 s^{7}+546 s^{6}+4536 s^{5}+22449 s^{4}+67284 s^{3}+118124 s^{2}+109584 s+40320}
$$

the numerator coefficients to make steady-state error zero. Therefore, the numerator polynomial obtained by CS is $N_{2}(s)=0.7515 s+2.5$

Finally, the transfer function of required second order reduced model is given as

$$
T_{2}(s)=\frac{0.7515 s+2.5}{s^{2}+3.5 s+2.5}
$$

The step responses of the original system $T_{4}(s)$, ROM with the proposed method $T_{2}(s)$, and ROM with the existing methods [32, 34, 43] are shown in figure 2 while the absolute error plot is given in figure 3. It is observed from the plots that, the proposed approach produces better results than the existing techniques $[32,34,43]$. The plot demonstrates that the response of the proposed ROM approximates the response of original system satisfactorily. Further, it is observed from absolute error curves that the absolute error obtained by the proposed method is lower in comparison with [32, 34, 43]. Table 1 represents the qualitative comparison of the proposed method with [32-34, 43, 45, 46] regarding time response parameters.
The poles of the given system are $a_{1}=-1.00$, $a_{2}=-2.00, \quad a_{3}=-3.00, \quad a_{4}=-4.00, \quad a_{5}=-5.00$, $a_{6}=-6.00, a_{7}=-7.00, a_{8}=-8.00$. By applying Eigen permutation, the poles of second order reduced model are obtained as $a_{e 1}=-1.00, a_{e 2}=-4.50$. Therefore, the denominator polynomial of reduced model is obtained as $D_{2}(s)=s^{2}+5.5 s+4.5$.

The CS optimization determines the coefficients of numerator polynomial with iter $=100$, and the transfer function is multiplied by a suitable scaling factor to have zero steady-state error. Finally, the required second order reduced model is obtained as

$$
T_{2}(s)=\frac{13.8931 s+4.5}{s^{2}+5.5 s+4.5}
$$

The step responses of the original system $T_{8}(s)$, second order reduced model with the proposed method $T_{2}(s)$, and the reduced model obtained by $[42,48]$ are plotted in figure 4 whereas plots of absolute error between higher order and reduced order models are shown in figure 5. The absolute error plots indicate that the absolute error obtained 
Table 1. Settling and rise time comparison for Example 1.

\begin{tabular}{lccc}
\hline Reduction method & Settling time & Rise time & Overshoot $(\%)$ \\
\hline Original system & 3.93 & 2.26 & 0 \\
Proposed method & 4.07 & 2.32 & 0 \\
Narwal and Prasad [43] & 3.16 & 2.15 & 1.39 \\
Desai and Prasad [34] & 3.62 & 2.28 & 0.274 \\
Philip and Pal [32] & 4.55 & 2.53 & 0 \\
Desai and Prasad [33] & 3.15 & 2.12 & 1.25 \\
Parmar et al [45] & 3.22 & 2.19 & 1.29 \\
Pal [46] & 5.57 & 2.2 & 2.69 \\
\hline
\end{tabular}

Table 2. Comparison between various reduced order algorithms for Example 1.

\begin{tabular}{lcccc}
\hline Reduction method & ISE $\left(\times 10^{-4}\right)$ & ITSE $\left(\times 10^{-4}\right)$ & IAE $\left(\times 10^{-2}\right)$ & ITAE $\left(\times 10^{-2}\right)$ \\
\hline Proposed method & 1.748 & 2.838 & 2.656 & 5.748 \\
Narwal and Prasad [43] & 19.91 & 79.95 & 11.05 & 45.93 \\
Desai and Prasad [34] & 2.84 & 10.09 & 4.44 & 17.377 \\
Philip and Pal [32] & 17.274 & 38.134 & 9.3144 & 25.526 \\
Desai and Prasad [33] & 19.455 & 76.188 & 11.122 & 45.368 \\
Parmar et al [45] & 18.487 & 69.771 & 11.194 & 43.906 \\
Pal [46] & 118.84 & 242.26 & 25.372 & 71.708 \\
\hline
\end{tabular}

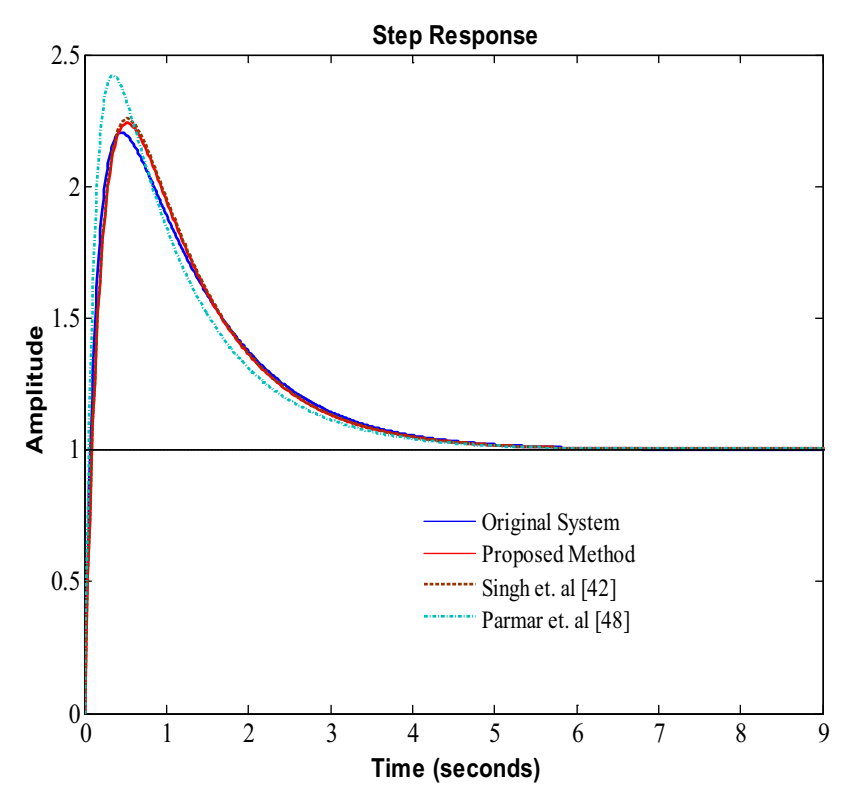

Figure 4. Step responses to different methods for Example 2.

by the proposed method is similar to [42] and comparatively better than [48]. The comparison between the proposed and the existing methods [42, 48-51] through performance indices and time response specifications are shown in tables 3 and 4, respectively. It is observed that the proposed approach gives comparatively satisfactory results.
Example 3: Consider a sixth order transfer function [42] given as

$\mathrm{T}_{6}(\mathrm{~s})=\frac{2 \mathrm{~s}^{5}+3 \mathrm{~s}^{4}+16 \mathrm{~s}^{3}+20 \mathrm{~s}^{2}+8 \mathrm{~s}+1}{2 \mathrm{~s}^{6}+33.6 \mathrm{~s}^{5}+155.9 \mathrm{~s}^{4}+209.5 \mathrm{~s}^{3}+102.4 \mathrm{~s}^{2}+18.3 \mathrm{~s}+1}$

The system has six poles located at $a_{1}=-0.1$, $a_{2}=-0.2, a_{3}=-0.5, a_{4}=-1, a_{5}=-5, a_{6}=-10$.

Now, the poles of second order reduced model by the Eigen permutation technique are obtained as $a_{e l}=-0.1$, $a_{e 2}=-1.68$. Therefore, the denominator polynomial of reduced model is $D_{2}(s)=s^{2}+1.78 s+0.168$

Now, the coefficients of numerator polynomial are computed by cuckoo search optimization algorithm and finally, the $2^{\text {nd }}$ order reduced model is given as

$$
T_{2}(s)=\frac{0.0818 s+0.168}{s^{2}+1.78 s+0.168}
$$

Figures 6 and 7 show the step responses and squared error plots of ROMs, respectively. Tables 5 and 6 provide the comparison through time response parameters and performance indices for ROMs by the proposed and the existing techniques $[42,47]$. The effectiveness of the proposed approach may easily be observed from the plots and tabular comparison.

Example 4: Consider the transfer function matrix of the $6^{\text {th }}$ order MIMO system [34, 43, 45] with two inputs and two outputs as 


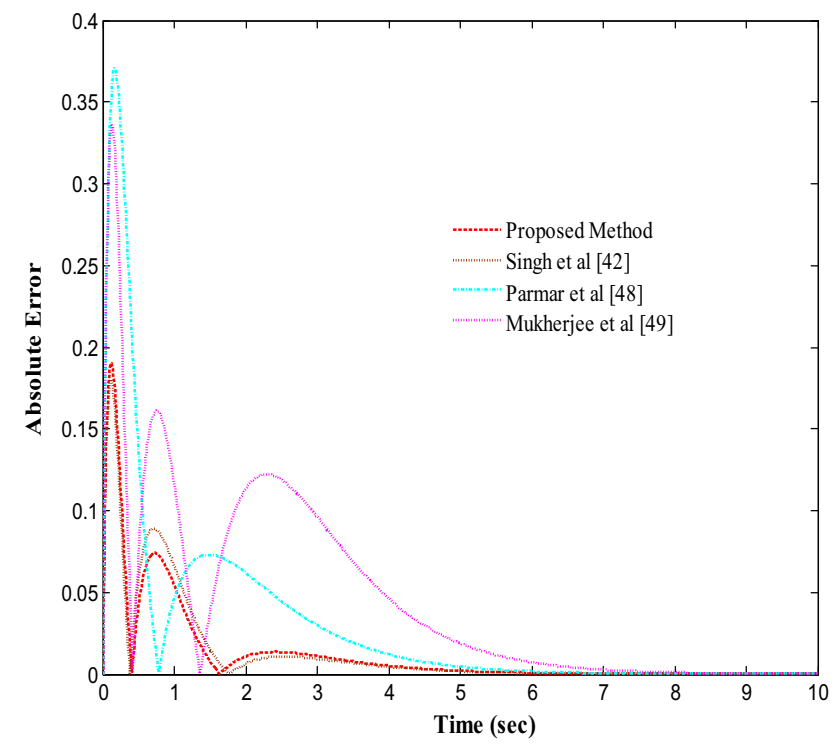

Figure 5. Plots of absolute error obtained by various methods for Example 2.

Table 3. Comparison between various reduced order algorithms for Example 2.

\begin{tabular}{lrrrr}
\hline Reduction method & \multicolumn{1}{c}{ ISE } & ITSE & IAE & ITAE \\
\hline Proposed method & $9.628 \times$ & $3.96 \times$ & 0.1236 & 0.141 \\
& $10^{-3}$ & $10^{-3}$ & & \\
Singh et al [42] & $1.008 \times$ & $4.77 \times$ & 0.128 & 0.138 \\
& $10^{-2}$ & $10^{-3}$ & & \\
Parmar et al [48] & $4.81 \times$ & $2.35 \times$ & 0.3007 & 0.389 \\
& $10^{-2}$ & $10^{-2}$ & & \\
Mukherjee et al [49] & $5.69 \times$ & $7.48 \times$ & 0.4574 & 0.948 \\
& $10^{-2}$ & $10^{-2}$ & & \\
Mittal et al [50] & $2.689 \times$ & $1.91 \times$ & 0.8098 & 1.210 \\
Mukherjee and Mishra & $2.689 \times$ & $1.91 \times$ & 0.8098 & 1.210 \\
[51] & $10^{-1}$ & $10^{-1}$ & & \\
\hline
\end{tabular}

Table 4. Settling and rise time comparison for Example 2.

\begin{tabular}{llcc}
\hline Reduction method & $\begin{array}{c}\text { Rise } \\
\text { time }\end{array}$ & $\begin{array}{c}\text { Settling } \\
\text { time }\end{array}$ & $\begin{array}{c}\text { Overshoot } \\
(\%)\end{array}$ \\
\hline Original system & 0.057 & 4.82 & 120 \\
Proposed method & 0.0717 & 4.68 & 124 \\
Singh et al [42] & 0.071 & 4.68 & 126 \\
Parmar et al [48] & 0.041 & 4.39 & 142 \\
Mukherjee et al [49] & 0.086 & 3.35 & 129 \\
Mittal et al [50] & 0.142 & 5.47 & 107 \\
Mukherjee and Mishra & 0.142 & 5.47 & 107 \\
$\quad$ [51] & & & \\
\hline
\end{tabular}

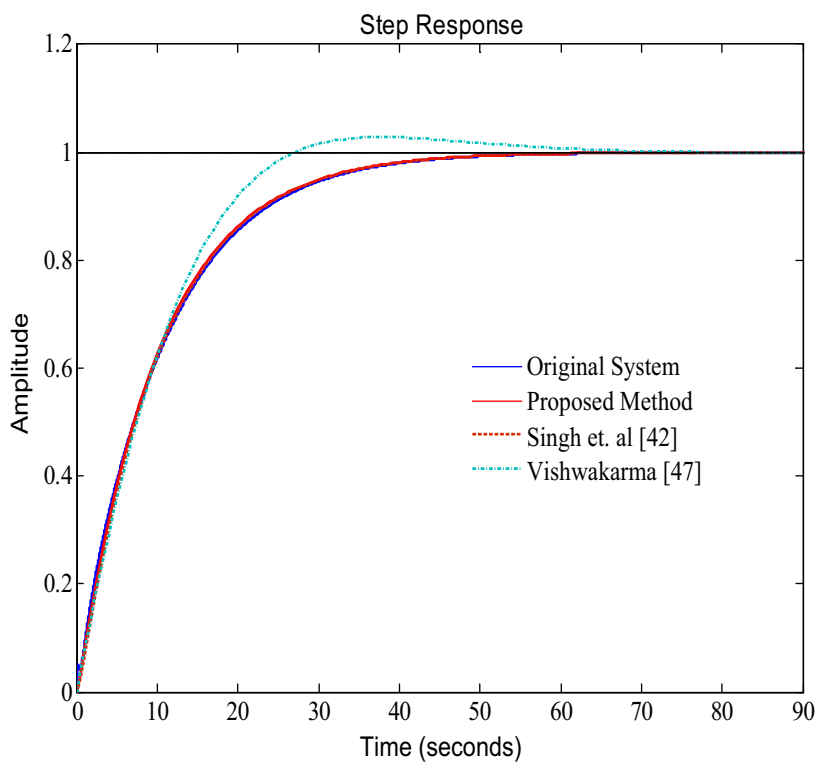

Figure 6. Step responses to different methods for Example 3.

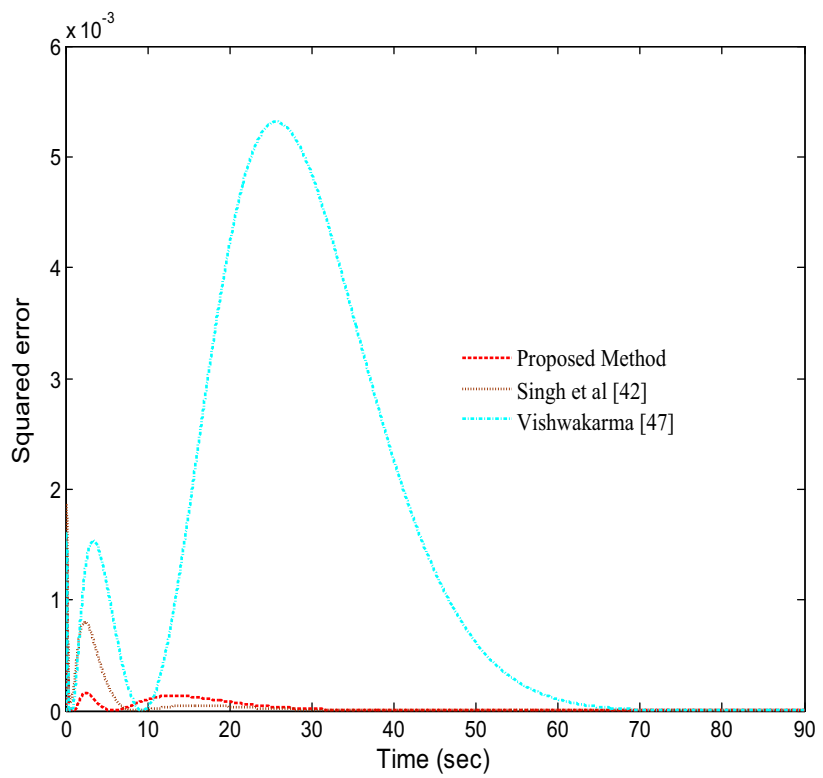

Figure 7. Plots of squared error obtained by various methods for Example 3.

Table 5. Settling and rise time comparison for Example 3.

\begin{tabular}{lccc}
\hline Reduction method & Rise time & Settling time & Overshoot $(\%)$ \\
\hline Original system & 22.7 & 40.1 & 0 \\
Proposed method & 22.0 & 39.2 & 0 \\
Singh et al [42] & 22.0 & 39.4 & 0 \\
Vishwakarma [47] & 17.7 & 47.8 & 2.81 \\
\hline
\end{tabular}


Table 6. Comparison between various reduced order algorithms for Example 3.

\begin{tabular}{lccr}
\hline Reduction method & ISE $\left(\times 10^{-3}\right)$ & ITSE $\left(\times 10^{-2}\right)$ & IAE $\left(\times 10^{-1}\right)$ \\
\hline Proposed method & 2.53 & 3.28 & 2.86 \\
Singh et al [42] & 3.68 & 2.10 & 2.79 \\
Vishwakarma [47] & 128 & 361 & 25.1 \\
\hline
\end{tabular}

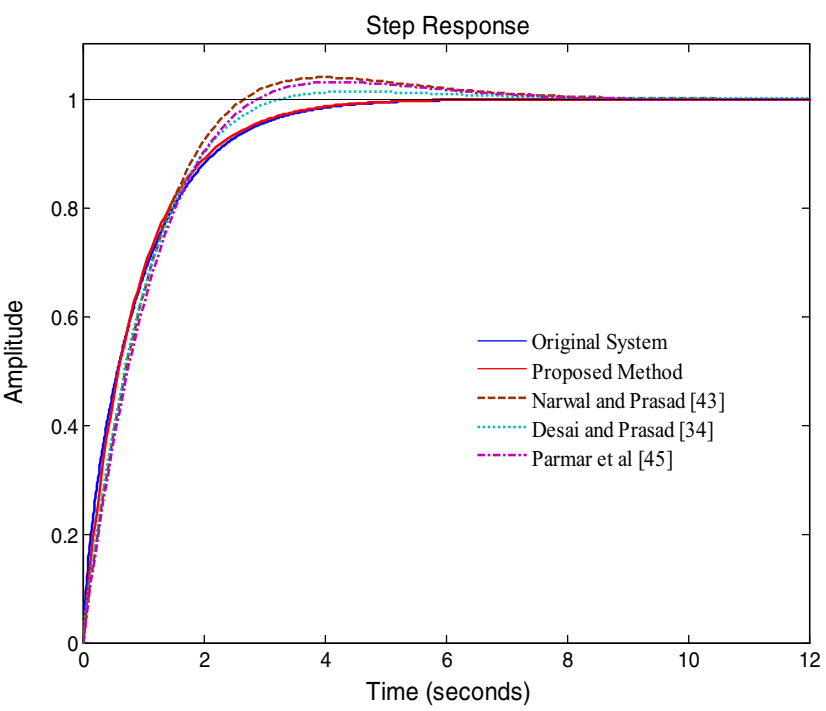

(a)

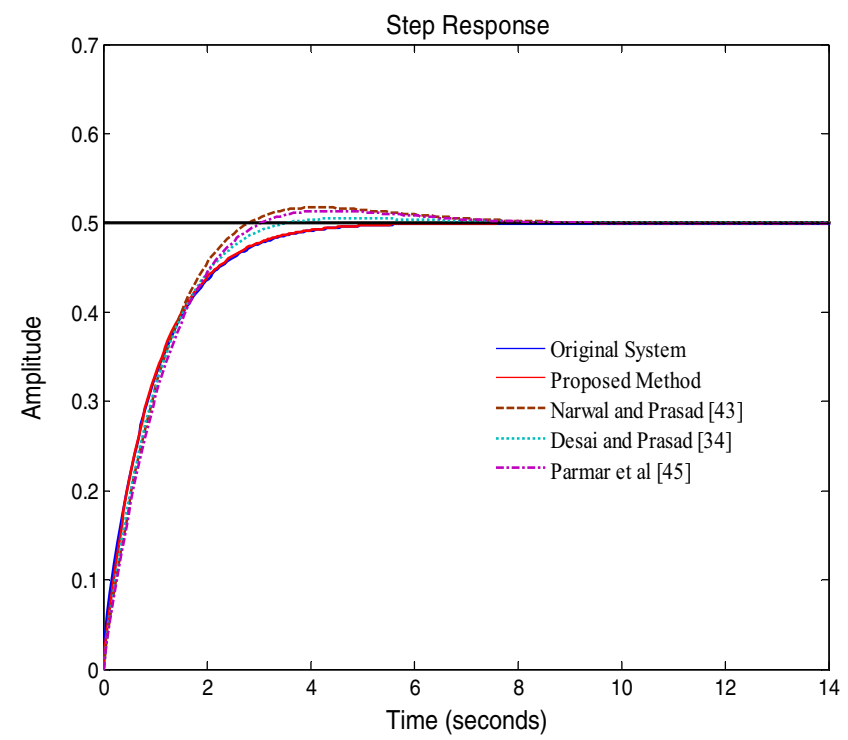

(c)

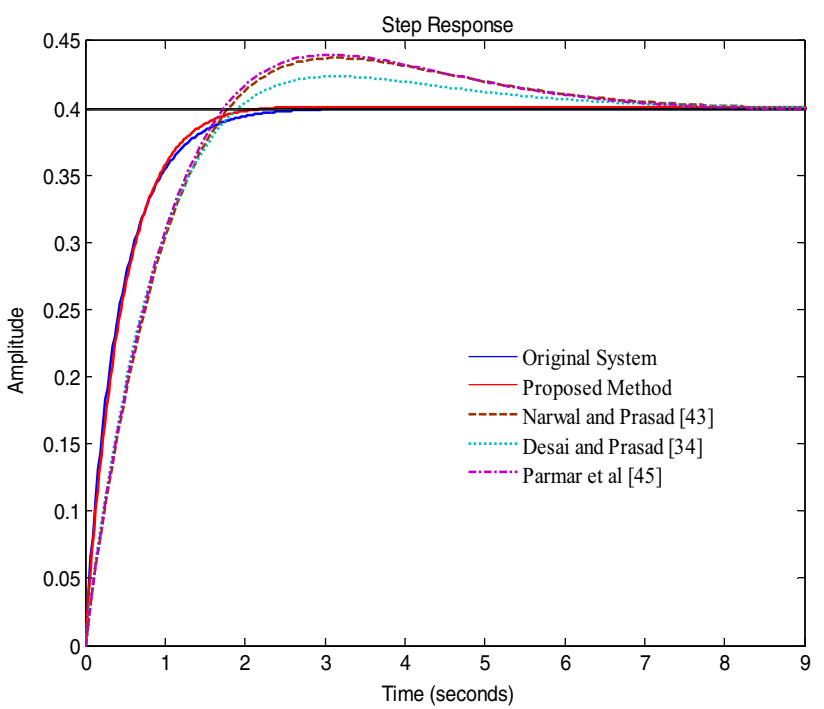

(b)

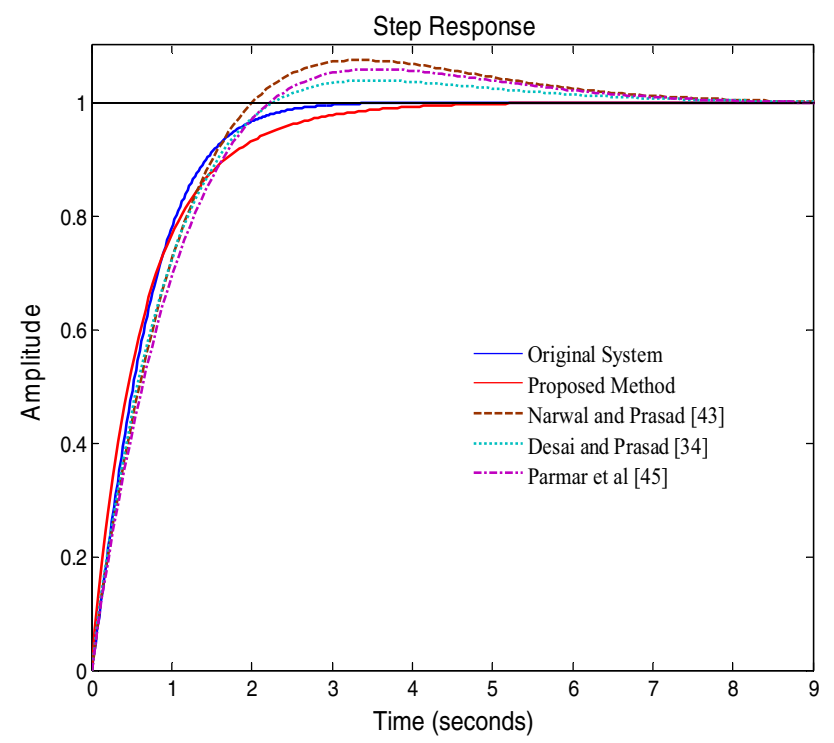

(d)

Figure 8. (a-d) Step responses of different methods for Example 4. 
Table 7. ISE Comparison between various reduced order algorithms for Example 4.

\begin{tabular}{lcccc}
\hline Reduction method & $\mathrm{R}_{11}$ & $\mathrm{R}_{12}$ & $\mathrm{R}_{21}$ & $\mathrm{R}_{22}$ \\
\hline Proposed method & $7.732 \times 10^{-4}$ & $7.690 \times 10^{-5}$ & $7.123 \times 10^{-5}$ & $3.55 \times 10^{-3}$ \\
Narwal and Prasad [43] & $1.62 \times 10^{-2}$ & $8.98 \times 10^{-3}$ & $2.97 \times 10^{-3}$ & $1.71 \times 10^{-2}$ \\
Desai and Prasad [34] & $7.026 \times 10^{-3}$ & $6.031 \times 10^{-3}$ & $1.024 \times 10^{-3}$ & $6.367 \times 10^{-3}$ \\
Parmar et al [45] & $1.45 \times 10^{-2}$ & $8.75 \times 10^{-3}$ & $2.539 \times 10^{-3}$ & $1.574 \times 10^{-2}$ \\
Prasad et al [52] & $3.069 \times 10^{-2}$ & $2.56 \times 10^{-3}$ & $2.62 \times 10^{-1}$ & $2.168 \times 10^{-2}$ \\
\hline
\end{tabular}

Table 8. Settling and rise time comparisons for Example 4.

\begin{tabular}{|c|c|c|c|c|c|c|}
\hline Reduction method & Rise time & Settling time & Overshoot (\%) & Rise time & Settling time & Overshoot $(\%)$ \\
\hline $\mathbf{R}_{11}$ & & & & \multicolumn{3}{|c|}{$\mathbf{R}_{12}$} \\
\hline Original system & 2.13 & 3.79 & 0 & 1.02 & 1.87 & 0 \\
\hline Proposed method & 2.01 & 3.68 & 0 & 0.98 & 1.63 & 0.237 \\
\hline Narwal and Prasad [43] & 1.76 & 5.94 & 3.92 & 1.29 & 6.35 & 9.33 \\
\hline Desai and Prasad [34] & 1.88 & 2.81 & 1.26 & 1.31 & 5.65 & 5.8 \\
\hline Parmar et al [45] & 1.98 & 5.82 & 3.28 & 1.34 & 6.38 & 10.1 \\
\hline Prasad et al [52] & 1.22 & 2.12 & 0 & 1.15 & 2.53 & 0 \\
\hline $\mathbf{R}_{21}$ & & & & \multicolumn{3}{|c|}{$\mathbf{R}_{22}$} \\
\hline Original system & 2.18 & 3.86 & 0 & 1.34 & 2.28 & 0 \\
\hline Proposed method & 2.11 & 3.8 & 0 & 1.61 & 3.13 & 0 \\
\hline Narwal and Prasad [43] & 1.84 & 5.85 & 3.44 & 1.41 & 6.26 & 7.4 \\
\hline Desai and Prasad [34] & 1.98 & 2.9 & 1.05 & 1.48 & 5.30 & 3.62 \\
\hline Parmar et al [45] & 1.94 & 5.7 & 2.85 & 1.52 & 6.17 & 6.01 \\
\hline Prasad et al [52] & 1.10 & 1.97 & 0 & 1.07 & 4.93 & 0 \\
\hline
\end{tabular}

$$
\begin{aligned}
T_{6}(s) & =\left[\begin{array}{ll}
\frac{2(s+5)}{(s+1)(s+10)} & \frac{(s+4)}{(s+2)(s+5)} \\
\frac{(s+10)}{(s+1)(s+20)} & \frac{(s+6)}{(s+2)(s+3)}
\end{array}\right] \\
& =\frac{1}{b_{6}(s)}\left[\begin{array}{ll}
a_{11}(s) & l a_{12}(s) \\
a_{21}(s) & a_{22}(s)
\end{array}\right]
\end{aligned}
$$

The denominator polynomial is given as $s^{6}+41 s^{5}+571 s^{4}+3491 s^{3}+10060 s^{2}+13100 s+6000$

Similarly, the numerator coefficients are

$$
\begin{aligned}
& a_{11}(s)=2 s^{5}+70 s^{4}+762 s^{3}+3610 s^{2}+7700 s+6000 \\
& a_{12}(s)=s^{5}+38 s^{4}+459 s^{3}+2182 s^{2}+4160 s+2400 \\
& a_{21}(s)=s^{5}+30 s^{4}+331 s^{3}+1650 s^{2}+3700 s+3000 \\
& a_{22}(s)=s^{5}+42 s^{4}+601 s^{3}+3660 s^{2}+9100 s+6000
\end{aligned}
$$

The system has six poles located at $a_{1}=-1, a_{2}=-2$, $a_{3}=-3, a_{4}=-5, a_{5}=-10, a_{6}=-20$.The poles of second order reduced model obtained by Eigen permutation are obtained as $a_{e 1}=-1.00, a_{e 2}=-2.05$. Hence, the denominator polynomial of ROM is given by

$$
D_{2}(s)=s^{2}+3.05 s+2.05
$$

Further, the numerator polynomial matrix of ROM by the $\mathrm{CS}$ algorithm is obtained as

$$
\left[\begin{array}{l}
N_{11}(s) N_{12}(s) \\
N_{21}(s) N_{22}(s)
\end{array}\right]=\left[\begin{array}{cc}
1.224 s+2.05 & 0.8597 s+0.82 \\
0.5550 s+1.025 & 1.5902 s+2.05
\end{array}\right]
$$

Therefore, the required transfer function of second order reduced model is

$$
\begin{aligned}
T_{2}(s)= & \frac{1}{D_{2}(s)}\left[\begin{array}{l}
N_{11}(s) N_{12}(s) \\
N_{21}(s) N_{22}(s)
\end{array}\right]=\frac{1}{\left(s^{2}+3.05 s+2.05\right)} \\
& {\left[\begin{array}{cc}
1.224 s+2.05 & 0.8597 s+0.82 \\
0.5550 s+1.025 & 1.5902 s+2.05
\end{array}\right] }
\end{aligned}
$$

The step responses of the original system, reduced models with the proposed and the existing methods $[34,43,45]$ are included in figure 8a-d. Further, a fruitful improvement in error index ISE validates the superiority of the proposed method over [34, 43, 45, 52], which is shown in table 7. The qualitative comparison of transient parameters of reduced models obtained by proposed and existing methods $[34,43,45,52]$ is given in table 8 . 


\section{Conclusion}

In this article, an improved mixed method is presented based on Eigen permutation and Cuckoo search optimization for reducing the order of complex SISO as well as MIMO continuous linear time-invariant systems. Eigen permutation approach determines the reduced order denominator polynomial; whereas cuckoo search algorithm is utilized to determine the coefficients of the numerator polynomial of reduced order model. The proposed approach guarantees the stability of the reduced model if the original higher order system is stable. The worthiness of the proposed method is verified by the numerical examples of SISO and MIMO systems and a significant improvement in the performance indices are obtained for resulting reduced order models. Further, this method can easily be extended to the approximation of discrete time systems also.

\section{References}

[1] Chen C F and Shich L S 1968 A novel approach to linear model simplification. Int. J. Control 8(6): 561-570

[2] Shamash Y 1976 Continued fraction methods for reduction of constant-linear multivariable systems. Int. J. Syst. Sci. 7(7): 743-758

[3] Chuang S C 1970 Application of continued-fraction method for modelling transfer functions to give more accurate initial transient response. Electron. Lett. 6(26): 861-863

[4] Shamash Y 1974 Stable reduced-order models using Padè type approximations. IEEE Trans. Autom. Control 19(5): 615-616

[5] Zakian V 1973 Simplification of linear time-invariant systems by moment approximants. Int. J. Control 18(3): 455-460

[6] Pal J 1983 Improved Padé approximants using stability equation method. Electron. Lett. 19(1): 426-427

[7] Shamash Y 1975 Linear system reduction using Padé approximation to allow retention of dominant modes. Int. J. Control 21(2): 257-272

[8] Lucas T N 1983 Factor division: a useful algorithm in model reduction. IEE Proc. 130(6): 362-364

[9] Wan B W 1981 Linear model reduction using Mihailov criterion and Padé approximation technique. Int. J. Control 33(6): 1073-1089

[10] Hutton M and Friedland B 1975 Routh approximations for reducing order of linear, time-invariant systems. IEEE Trans. Autom. Control AC 20(3): 329-337

[11] Appiah R K 1978 Linear model reduction using Hurwitz polynomial approximation. Int. J. Control 28(3): 477-488

[12] Appiah R K 1979 Padè methods of Hurwitz polynomial approximation with application to linear system reduction. Int. J. Control 29(1): 39-48

[13] Chen T C, Chang C Y and Han K W 1980 Stable reducedorder Padè approximants using stability-equation method. Electron. Lett. 16(9): 345-346

[14] Gutman P O, Mannerfelt C F and Molander P 1982 Contributions to the model reduction problem. IEEE Trans. Autom. Control 27(2): 454-455
[15] Yadav J S, Patidar N P, Singhai J, Panda S and Ardil C 2011 A combined conventional and differential evolution method for model order reduction. Int. J. Electr. Comput. Energ. Electron. Commun. Eng. 5(9): 1284-1291

[16] Panda S, Tomar S K, Prasad R and Ardil C 2009 Model reduction of linear systems by conventional and evolutionary techniques. Int. J. Electr. Comput. Energ. Electron. Commun. Eng. 3(11): 2150-2156

[17] Liaw C M 1989 Mixed method of model reduction for linear multivariable systems. Int. J. Syst. Sci. 20(11): 2029-2041

[18] Narwal A and Prasad R 2015 A novel order reduction approach for LTI systems using Cuckoo search and Routh Approximation. In: IEEE International Advanced Computing Conference, pp. 564-569

[19] Pal J 1980 System reduction by a mixed method. IEEE Trans. Autom. Control AC 25(5): 973-976

[20] Pal J, Sinha A K and Sinha N K 1995 Reduced order modelling using pole clustering and time moment matching. $J$. Inst. Eng. (India) Electr. Eng. Div. 76: 1-6

[21] Parmar G, Prasad R and Mukherjee S 2007 A mixed method for large-scale systems modelling using eigen spectrum analysis and Cauer second form. IETE J. Res. 53(2): 93-102

[22] Sikander A and Prasad R 2015 Linear time invariant system reduction using a mixed methods approach. Appl. Math. Model. 39(16): 4848-4858

[23] Singh J, Vishwakarma C B and Chatterjee K 2016 Biased reduction method by combining improved modified pole clustering and improved Pade approximants. Appl. Math. Model. 40: 1418-1426

[24] Wilson D A 1970 Optimal solution of model reduction problem. Proc. IEE 117(6): 1161-1165

[25] Wilson D A 1974 Model reduction for multivariable systems. Int. J. Control 20(1): 57-64

[26] Goldberg D E 1989 Genetic Algorithms in Search, Optimization, and Machine Learning. Boston, MA, USA: Addison-Wesley Longman Publishing Co. Inc.

[27] Satakshi, Mukherjee S and Mittal R C 2005 Order reduction of linear discrete systems using a genetic algorithm. Appl. Math. Model. 29(6): 565-578

[28] Soloklo H N and Farsangi M M 2013 Chebyshev rational functions approximation for model order reduction using harmony search. Sci. Iran. 20(3): 771-777

[29] Kennedy J and Eberhart R 1995 Particle swarm optimization. IEEE Int. Conf. Neural Netw. 4: 1942-1948

[30] Sikander A and Prasad R 2015 Soft computing approach for model order reduction of linear time invariant systems. Circuits Syst. Signal Process. 34: 3471-3487

[31] Gallehdari Z, Karrari M and Malik O P 2009 Model order reduction using PSO algorithm and it's application to power systems. In: IEEE International Conference on Electric Power and Energy Conversion Systems EPECS '09, pp. 1-5, Sharjah

[32] Philip B and Pal J 2010 An evolutionary computation based approach for reduced order modelling of linear systems. In: IEEE International Conference on Computational Intelligence and Computing Research (ICCIC) Coimbatore, India, pp. 28-29

[33] Desai S R and Prasad R 2013 A new approach to order reduction using stability equation and big bang big crunch optimization. Syst. Sci. Control Eng. 1(1): 20-27

[34] Desai S R and Prasad R 2013 A novel order diminution of LTI systems using Big Bang Big Crunch optimization and 
Routh Approximation. Appl. Math. Model. 37(16-17): 8016-8028

[35] Biradar S, Hote Y V and Saxena S 2016 Reduced-order modeling of linear time invariant systems using big bang big crunch optimization and time moment matching method. Appl. Math. Model. 40(15-16): 7225-7244

[36] Yang X S and Deb S 2010 Engineering optimization by Cuckoo Search. Int. J. Math. Model. Numer. Optim. 1(4): 330-343

[37] Long W, Liang X, Huang Y and Chen Y 2014 An effective hybrid cuckoo search algorithm for constrained global optimization. Neural Comput. Appl. 25: 911-926. https://doi.org/ 10.1007/s00521-014-1577-1

[38] Kanagaraj G, Ponnambalam S G and Jawahar N 2013 A hybrid cuckoo search and genetic algorithm for reliabilityredundancy allocation problems. Comput. Ind. Eng. 66: 1115-1124

[39] Sikander A, Thakur P and Uniyal I 2016 Hybrid method of reduced order modelling for LTI system using evolutionary algorithm In: 2nd International Conference on Next Generation Computing Technologies (NGCT), Dehradun, pp. $84-88$

[40] Sikander A and Thakur P Reduced order modelling of linear time-invariant system using modified cuckoo search algorithm. Soft Comput. https://doi.org/10.1007/s00500-0172589-4

[41] Walton S, Hassan O and Morgan K 2013 Reduced order mesh optimisation using proper orthogonal decomposition and a modified cuckoo search. Int. J. Numer. Meth. Eng. 93: $527-550$

[42] Singh J, Chatterjee K and Vishwakarma C B 2014 System reduction by eigen permutation algorithm and improved Padé approximations. Int. J. Math. Comput. Phys. Electr. Comput. Eng. 8(1): 180-184

[43] Narwal A and Prasad R 2015 A novel order reduction approach for LTI systems using cuckoo search optimization and stability equation. IETE J. Res. 62(2): 1-10

[44] Yang X S and Deb S 2010 Nature-Inspired Meta Heuristic Algorithms. Second Edition UK: Luniver Press, pp. 105-117

[45] Parmar G, Prasad R and Mukherjee S 2007 Order reduction of linear dynamic systems using stability equation method and GA. Int. J. Electr. Comput. Energ. Electron. Commun. Eng. 1(2): 236-242

[46] Pal J 1979 Stable reduced order Padé approximants using the Routh Hurwitz array. Electron. Lett. 15(8): 225-226

[47] Vishwakarma C B 2009 Model Order Reduction Using of Linear Dynamic Systems for Control System Design Ph.D. Thesis IIT Roorkee, Roorkee, India

[48] Parmar G, Prasad R and Mukherjee S 2007 System reduction using factor division algorithm and eigen spectrum analysis. Appl. Math. Model. 31(11): 2542-2552

[49] Mukherjee S, Satakshi and Mittal R C 2005 Model order reduction using response matching technique. J. Frankl. Inst. 342(5): 503-519

[50] Mittal A K, Prasad R and Sharma S P 2004 Reduction of linear dynamic systems using an error minimization technique. J. Inst. Eng. India Part El Electr. Eng. Div. 84: 201-206

[51] Mukherjee S and Mishra R N 1987 Order reduction of linear systems using an error minimization technique. J. Frank. Inst. 323(1): 23-32

[52] Prasad R, Pal J and Pant A K 1995 Multivariable system approximation using polynomial derivatives. J. Inst. Eng. India Part El Electr. Eng. Div. 76: 186-188 